\title{
Cryptocurrency Business Covers the Psychological Impact of the Business Market on the Youth Segment
}

\author{
Mattie Philavong ${ }^{1}$, Solasak Phomvongsa ${ }^{1}$ \\ ${ }^{1}$ Faculty of Economics and Business Management, National University of Laos \\ Received: February 13, 2021 \\ Revised: March 13, 2021 \\ Accepted: March 27, 2021
}

\begin{abstract}
In comparison to the production of physical currency, e-money is a commodity that is provided by banks or financial-sector firms, such as paypal. Then the transaction and investment processes shifted with technical advancements. Today, cryptocurrencies seem to render commerce and transaction structures simpler. The invention of electronic money would help young people expand, given the universal usage of transaction resources to promote goods owned by business owners in all international countries. The blockchain mechanism will operate separately, apart from the transaction speed, without any agency regulating or manipulating all continuing transactions.
\end{abstract}

Keywords: Cryptocurrency, Technology, Transactions \& Youth

\section{Introduction}

The instruments of payment transfers have witnessed various innovations today. You can purchase goods on the Internet without using paper money. You can order them. Cryptocurrency is one of the digital money assets to address modern online financial transactions' needs (Chuen et al., 2017). Cryptocurrency is a digitally recognised commodity as digital money (Liang et al., 2019). This is very distinct from the standard money, where crypto-monetary devices are used for virtual internet network transfers. This currency is autonomous, meaning that no entity is a transaction broker. Payments are thus carried out on a peer-to-peer basis, that is to say directly from the sender to the recipient.

Furthermore, all transactions are documented with optimum protection via an established method. Because the cryptocurrency is decentral, sophisticated and computer-competent requirements are essential. Typically a blockchain network is used to allow transactions. The cryptocurrency theory is the same as the economic principle, that the price can rise while demand is large (Iwamura et al., 2019; Al-Naji et al., 2017; Fantacci, 2019).

The more people buy, the higher the price. This expenditure is, however, included in the definition of elevated risk. At the moment, several businesses or organisations, including hotels, flights, restaurants and apps, have introduced payment instruments utilizing virtual currency. At least two firms, Overstock and Newegg, have been utilizing this transaction mechanism. Most businesses allow only Bitcoins, though. This method makes it possible for business people to operate without the need for cash on the young business sector. In today's days, young people play the right part in enhancing company efficiency by enjoying exceptional times.

\section{Cryptocurrency Development}

Most citizens have known what blockchain is. The central government, however, firmly opposed the presence of this digital currency. It is not in reality accepted as a legitimate trade or payment tool in the country under constitutional provisions. 
The acceptance of payment instruments is only in rupiah currency. However, it is still permitted and lawful to live $\mathrm{n}$ cryptocurrency.

The Bank offers tips to save and purchase and sell trades with your own expense as assets. Multiple means of utilizing online payment methods in cryptocurrencies. Of course you will use the virtual money for different forms of purchases if you use the cryptocurrency daily. The trade of the traditional currency with bitcoin begins from capital market operations. Then personal expenses have been provided in digital form by the corporation where you transact.

So large financing or investment would reduce transaction costs if the supported project fails (Gerber et al., 2012; Pierrakis \& Collins, 2013; Solomon et al., 2015). In America, there are cryptocurrency trading markets such as the ICE, CME (Chicago merchant exchange) and CBOE (Chicago Board Option Exchange). Cryptocurrency comes from well encrypted and special cryptography. You ought to remember, the valuation of each kind of cryptocurrency is identical to a financial asset, where the cryptocurrency value can rise as demand is strong enough for a few miners.

That implies that cryptocurrency currency value is unpredictable and may rise or decline on the basis of consumer availability or confidence. Today's top classification of cryptocurrency is bitcoin and Etherium.

E-money is an automated commodity generated by banks or financial sector firms such as paypal with the production of paper money. Then the transaction and investment processes shifted with technical advancements. Today, cryptocurrencies seem to render commerce and transaction structures simpler. The usage of actual currency (fiatcurrency) is deemed to have the power of cryptocurrencies (Claeys et al., 2018; Sontakke \& Ghaisas, 2017). Cryptocurrency benefits in terms of transmission pace do not impact nation boundaries. This method is very useful, as opposed to fiat currencies. With high versatility, this digital money is optimistic to enter the planet with a currency that will eliminate limits that cannot be exceeded. So while you are overseas, it can be used and transported. In a country which legalizes Bitcoin as a legal means of payment, for example, like Japan, an individual does not have to exchange his money for yen. So transfers are simpler to do. The blockchain mechanism will operate separately, apart from the transaction speed, without any agency regulating or manipulating all continuing transactions. This device is still very stable from cyber threats, since all details available are shared by all machine miners in the world. This system is not able to interfere with transactions. This is what distinguishes them from fiat-development that involves a body or bank to oversee the operation.

Since this invention cannot be regulated by anybody. The price of the cryptocurrency is thus puraly dictated by the demand and delivery such that uncertainty levels or the rate of changes in value are quite large, therefore it fluctuates according to consumer demand and availability. However, whatever the nature of the digital currency, the currency of a nation would not be able to substitute it, because the currency is the identity of that country. Therefore, the purpose of the digital currency is only to make things easy for citizens who sometimes visit numerous countries, so that purchases in visited countries are not too complicated. Digital currencies (CCMs) are being used by more and more citizens from many developing countries and different regulations have culminated in a dramatic growth in digital currencies' value. This opens up new possibilities for investments, leading more consumers to begin buying crypto assets like Bitcoin and other other coins to participate in prices moving the digital currency. But the gamble is not risk-free to invest in the digital money, since the risk is higher than all types of investing, but still implies great returns. This is because the movements of this digital money cannot be regulated by either government or corporation. It is the supply and demand 
on the market that purely determines the flow of the digital currency. Moreover, without a proper investment deal, investors are susceptible to deception or scam. To date, the institution of the Financial Authority, has stressed that the industry of cryptocurrencies does not have approval and it has no regulatory mechanism for such an investment. The more risky blockchain trade, so this brings a greater danger.

Moreover, several digital currency providers are not a marketplace but guarantee high returns in the purchase of their goods. With respect to the prevalent illicit investment in cryptomonetary goods, the ICTF called on the public to know the forms in which the criminals gave themselves. Previously, financial companies have stressed that digital or cryptocurencies, such as bitcoin, would not be used or made possible by the Financial Services Authority. In the law of the Republic of No 7 of 2011 on currencies which enable rupiah to be the only legal means of payment, the restriction of use of cryptocurrency is controlled.

However, the authority's appeal has little impact on Crypto-monetary investment. As one of the largest cryptocurrency exchanges in Southeast Asia, Digital Asset Exchange (INDODAX) reported that the number of investors registering on its website has surpassed 1.1 million investors, with an average of some three thousand new investors every day. The number of contributors, including 1,18 million investors, has almost corresponded to the number of participants. In comparison to the high growth in investors every day.

Indodax also has a rather large transaction volume, at 10 billion US dollars per day in the movement of crypto currency. As cryptocurrency values rise, the amount will double. However, this amount is far less than the transaction value of 7,56 billion a day on the stock exchange. You may claim it is not really relevant to the Authority's attractiveness to the culture. Many things, internal and external influences, will affect an individual's investment decision. The decision-making philosophy is focused on the principle of happiness, which enables people to decide whether efficiency increases or decreases to improve satisfaction. This definition is used to optimize the usefulness of each human effort to obtain fulfillment. Investor decisions can therefore be made rationally to optimize the value of investment decision-making for customers.

The investment judgment and the outcomes to be obtained are affected by many psychological variables. Consequently, investment analyzes utilizing psychology and financial analysis are regarded as financial behavior (Behavior Finance). In decision-making philosophy, the principle of rational investors assumes the selected behavior produces the maximum anticipated benefit in decision-making. In reality, we also notice that people are unreasonable in their predictions and making systemic mistakes. Financial players now understand that people will conclude irrationally. Informational errors may impair returns on investments, which will in turn impact on investors' equity.

While they have become specialists in modern investing book theory, investors would not spend if the decision-making process is strongly motivated by psychological prejudiciation. Some of the psychological prejudices which may have an effect on a person's investment choices are influence perceptions that can lead a person to get interested in an illusion that can make him/her make the wrong decision in making the correct investment. Illusions of power that lead an individual to feel capable of solving challenges, even though the reverse is valid, Motivation is one of the psychological causes of an individual, in addition to power delusions, strong motivation increases the psychological degree and makes the choice of a motivating expenditure possible for a person to spend more objectively. 


\section{The Advantages and Disadvantages of Cryptocurrency for the Young Business Market}

Next is the last material regarding the advantages and disadvantages of virtual currency. Where everyone can use cryptocurrency without binding regulations and conditions. With digital currency, every user can see various transaction activities that have been carried out. Of course, this transparency also has a limit where you cannot see the person carrying out the transaction. That is, each user or user will be responsible for their respective currencies. Finally, transactions using virtual currency are very fast when compared to transactions through banks. Some countries still do not allow this currency and it is still considered illegal. So, for some countries, cryptocurrency is not valid as a legal online payment tool.

Many people use cryptocurrency for criminal purposes. They can carry out illegal transactions without being noticed by exploiting loopholes in cryptographic code. If you do not memorize or forget your password, it will be very risky to lose money on the account that was created. Before making a decision to invest, an investor must understand the relationship between the risk and return of an investment, usually the greater the risk level of an investment, the greater the return to be obtained and vice versa, because the relationship between risk and return of an investment has a relationship. which is linear.

Decisions concerning the allocation of funds originating from within as well as funds originating from outside the company in various forms of investment. Return is the return on the yield on securities or investment which is usually expressed in the form of a percentage rate. The benefits that investors enjoy on the investment they make. Investors aim to invest is to get a return or profit. But in reality, the rate of return on returns that investors actually get (actual return) is not always the same as the rate of return that was previously expected (expected return). In other words, investors who invest face the risk of the possibility of deviating the true rate of return from the expected rate of return.

Thus, in investing in addition to paying attention to the rate of return, investors must always consider the risk level of an investment. unpleasant consequences (detrimental, harmful) from an act or action. the deviation (variability) between the expected return and the actual return. investment risk as an advantage that deviates from the expected. The deviation is linear and directly proportional, the greater the deviation between the level of actual profit and the expected rate of return, which means the greater the risk to be faced. Thus it can be understood that risk is the possibility of an actual return that is different from the expected return. And both risk and return have a linear relationship, the greater the risk of an asset, the greater the expected return on the asset, and vice versa.

\section{Conclusion}

Cryptocurrency is a digital asset in the form of digital currency as a transaction for buying and selling products or services through an internet network-based computer device. There are many different types of digital currencies, including bitcoin, litecoin, dogecoin, feathercoin, and bitcoincash. The function of cryptocurrency is to carry out buying and selling transactions online, doing mining, and as a means for investment. Some advantages and disadvantages for the youth business market It is universal, everyone can use cryptocurrency without binding regulations and any conditions, Transparent, Have control over personal, Fast and accurate. Meanwhile, there are some disadvantages of using virtual currency. Not fully licensed, In some countries this currency is still not allowed and it is still considered illegal. So, for some countries, cryptocurrency does not act as a legal means of online payment, opening security gaps and password systems. If you do not memorize or forget your password, it will be very risky to lose money on the account that has been created. 


\section{References}

Al-Naji, N., Chen, J., \& Diao, L. (2017). Basis: a price-stable cryptocurrency with an algorithmic central bank. Basis. io.

Chuen, D. L. K., Guo, L., \& Wang, Y. (2017). Cryptocurrency: A new investment opportunity?. The Journal of Alternative Investments, 20(3), 16-40.

Claeys, G., Demertzis, M., \& Efstathiou, K. (2018). Cryptocurrencies and monetary policy (No. 2018/10). Bruegel Policy Contribution.

Fantacci, L. (2019). Cryptocurrencies and the Denationalization of Money. International Journal of Political Economy, 48(2), 105-126.

Gerber, E. M., Hui, J. S., \& Kuo, P. Y. (2012). Crowdfunding: Why people are motivated to post and fund projects on crowdfunding platforms. In Proceedings of the international workshop on design, influence, and social technologies: techniques, impacts and ethics (Vol. 2, No. 11, p. 10).

Iwamura, M., Kitamura, Y., Matsumoto, T., \& Saito, K. (2019). Can we stabilize the price of a cryptocurrency?: Understanding the design of Bitcoin and its potential to compete with Central Bank money. Hitotsubashi Journal of Economics, 41-60.

Liang, J., Li, L., Chen, W., \& Zeng, D. (2019). Towards an understanding of cryptocurrency: a comparative analysis of cryptocurrency, foreign exchange, and stock. In 2019 IEEE International Conference on Intelligence and Security Informatics (ISI) (pp. 137139). IEEE.

Pierrakis, Y., \& Collins, L. (2013). Crowdfunding: A new innovative model of providing funding to projects and businesses. Available at SSRN 2395226.

Solomon, J., Ma, W., \& Wash, R. (2015). Don't wait! How timing affects coordination of crowdfunding donations. In Proceedings of the 18th acm conference on computer supported cooperative work \& social computing (pp. 547-556).

Sontakke, K. A., \& Ghaisas, A. (2017). Cryptocurrencies: A Developing Asset Class. International Journal of Business Insights \& Transformation, 10(2). 